\title{
Detección de infracciones y matrículas en motocicletas, mediante visión artificial, aplicado a Sistemas Inteligentes de Transporte
}

\author{
Jesús Valencia ${ }^{1}$, Tomas Ramirez-Guerrero ${ }^{1}$, Leonel Castañeda ${ }^{1}$, Mauricio Toro ${ }^{1,2}$ \\ inv_jvalencia@eafit.edu.co, teramirezg@eafit.edu.co, lcasta@eafit.edu.co,mtorobe@eafit.edu.co \\ ${ }^{1}$ Universidad EAFIT, Escuela de Ingeniería, Grupo de Estudios en Mantenimiento (GEMI), Medellín, \\ Colombia. \\ ${ }^{2}$ Universidad EAFIT, Escuela de Ingeniería, Grupo I+D+I en Tecnologías de la Información y las \\ Comunicaciones (GIDITIC), Medellín, Colombia.
}

DOI: 10.17013/risti.37.1-15

Resumen: El funcionamiento incompleto de los sistemas de transporte ha propiciado el incremento del transporte ilegal de pasajeros mediante motocicletas a nivel mundial, causando altos índices de accidentalidad. En las ciudades intermedias de Colombia, el principal método de control aplicado al transporte informal consiste en imponer multas a los infractores identificados por las autoridades de tránsito. Este artículo propone el desarrollo de una aplicación que, mediante técnicas de visión artificial, sirva como herramienta para los oficiales de tránsito en la detección de tres tipos de infracciones cometidas por motociclistas: no usar casco de protección; circular por zonas prohibidas; transportar parrillero en lugares donde no está permitido. Nuestro caso de estudio fue la ciudad de Valledupar, donde se tomaron 105 imágenes de motociclistas mientras conducían. Los resultados muestran una precisión del 87,5\% en la detección de infracciones, mostrando la pertinencia de la aplicación como herramienta auxiliar para desincentivar el transporte informal.

Palabras-clave: Transporte público; Detección de objetos; AI; OCR; Aplicaciones móviles.

\section{Detection of infractions and number-plates motorcycles, through artificial vision, in Intelligent Transportation Systems}

\begin{abstract}
Incomplete operation of transportation systems has led to an increase in illegal passenger transportation, by the means of motorcycles, at worldwide, causing high accident rates. In the intermediate cities of Colombia, the main control method applied to informal transportation is to impose fines to identified offenders by transit authorities. This work proposes the development of an application that, by means of computer vision, serves as a tool for transit officers in the detections of three infringements types committed by motorcyclists: not wearing a helmet; circulate in prohibited areas; transporting passenger in sites where it's not allowed.
\end{abstract}


Our case study was Valledupar city, where 105 motorcyclists' images were taken while they are driving. Results show an accuracy of $87,5 \%$ in infringements detection, showing the relevance of this application as an assistance tool to disincentive informal transportation.

Keywords: Public transportation; Object detection; AI; OCR; Mobile applications.

\section{Introducción}

La motocicleta se ha convertido en uno de los principales medios de transporte en Tailandia, India, Brasil y Colombia. Para el año 2016, en India fueron vendidas un total de 17.7 millones de motocicletas (Doval, 2017). El Estudio en Profundidad de Accidentes de Motocicleta, realizado en cinco países europeos, menciona que en el $68,7 \%$ de los incidentes reportados, el uso del casco ha prevenido o reducido el traumatismo craneoencefálico sufrido por el motociclista; por tal razón, en muchos países la legislación hace obligatorio el uso del casco por parte de los conductores de motocicletas (Association des Constructeurs Européens de Motocycles [ACEM], 2009).

Aunque la legislación en diversos países exige el uso obligatorio del casco de seguridad, muchos motociclistas no lo portan. Esto disminuye la seguridad vial, por lo que ha motivado a los investigadores a crear soluciones que ayuden a las autoridades de tránsito a contrarrestar dichas infracciones. En India se realizó una investigación cuyo objetivo fue desarrollar un sistema automatizado para distinguir el porte de cascos en motociclistas e imponer multas a los infractores como lo indica la ley, a fin de brindar una herramienta de apoyo para el departamento de policía (Rohith et al., 2019). En Tailandia se desarrolló un sistema para detectar automáticamente los motociclistas y determinar si están usando cascos de seguridad; el sistema extrae objetos en movimiento y los clasifica como motocicleta u otro tipo de objeto, basado en características tomadas de las imágenes y usando algoritmos de aprendizaje profundo (Waranusast et al., 2013). En Brasil se creó una metodología de visión artificial para detectar motociclistas sin casco mediante una estrategia de dos etapas: la detección de motocicletas y la detección del uso del casco (Silva et al., 2014). Los trabajos anteriores son pertinentes en lugares donde el objetivo es mejorar la vigilancia en las carreteras principales, el uso de casco sea obligatorio, el trabajo de control para el uso reglamentario del casco sea intensivo -como es el caso de Colombia; lugares donde es evidente mejorar la aplicación de las leyes de tránsito, particularmente para infracciones donde no existen métodos de detección automática.

La falta de un sistema de transporte público que preste dicho servicio con factores de calidad tales como cobertura, eficiencia, accesibilidad, y sostenibilidad, es una de las principales causas del auge del transporte informal e ilegal de pasajeros en varias ciudades de Colombia (Ministerio de Transporte, 2018a). Gwilliam (2002) establece que el transporte informal es un servicio de traslado de pasajeros disponible al público, el cual está fuera del sistema regulador tradicional de transporte público. En Colombia, el surgimiento de diversas modalidades de transporte informal se relaciona con la eliminación de rutas de transporte público colectivo, lo cual ocasionó un déficit en cobertura que fue aprovechado por los transportadores informales como oportunidad de mercado para ofrecer sus servicios (Tobón y Galvis, 2009). 
El uso de alternativas informales de transporte-en especial las que emplean motocicletas, como el "mototaxismo" - han causado una disminución considerable de la seguridad vial en Colombia. Según cifras de la Agencia Nacional de Seguridad Vial (ANSV), en el año 2018 fallecieron más de tres mil personas en accidentes de tránsito que involucraron motocicletas; de acuerdo con esto, el Ministerio de Transporte afirma que existe una correlación trágica entre la ilegalidad y la siniestralidad vial (Observatorio Nacional de Seguridad Vial, 2019).

El método principal para contrarrestar el transporte informal empleado por las autoridades de tránsito locales y nacionales consiste en la imposición de multas por infracciones relacionadas con esta actividad, tales como: no usar el casco y los elementos de protección; circular por zonas prohibidas y durante días restringidos; transportar parrillero en ciudades donde no está permitido. Dicho método de control a los motociclistas que ejercen transporte informal es ineficiente; esto se debe a la poca fuerza policial presente en los municipios donde tiene más arraigo este problema, a lo cual se suma las limitaciones propias de los sentidos humanos.

Las medidas restrictivas son establecidas, en primer lugar, por los mandatarios locales de cada municipio, ya que estas sirven como base a los oficiales de tránsito para ejercer control vial. Tal como es el caso de la ciudad de Valledupar, Colombia, donde la alcaldía municipal decretó medidas de control como las siguientes: restringir la circulación de motocicletas en los días hábiles de la semana, dentro de la zona céntrica de la ciudad; restringir la circulación de todo tipo de motocicletas el día miércoles de cada semana, en todo el perímetro urbano, entre las 08:0o y las 18:0o horas; prohibir el transporte de parrillero en motocicleta, en toda la ciudad, el día sábado de cada semana, entre las 07:0o y las 19:00; reglamentar el uso obligatorio del casco tanto para el conductor como el parrillero (Alcaldía de Valledupar, 2018). Estas disposiciones, que son permitidas en otras ciudades, son convertidas en infracciones sancionables para el control del transporte informal.

Por lo tanto, la hipótesis de este artículo es la siguiente: usando una aplicación móvil ¿es posible detectar el número de matrícula y las infracciones de tránsito hechas por los conductores de motocicletas, manteniendo un alto nivel de precisión y exactitud? El sistema propuesto en este trabajo sirve como herramienta de apoyo para los oficiales de tránsito encargados del monitoreo de las motocicletas en la ciudad. La aplicación permite capturar en flagrancia a los infractores, de forma automática, que circulen por un puesto de control ubicado por los oficiales de tránsito. Esto es posible gracias al uso de modelos entrenados de aprendizaje automático y visión artificial, los cuales fueron adaptados para la detección de tres tipos de infracciones: no usar el casco de protección; circular en zonas y durante días prohibidos; circular con parrillero cuando la normatividad municipal no lo permite. Es de resaltar que los resultados obtenidos son la continuación del trabajo Automatic detection of number-plate and traffic infractions of motorcyclists by Intelligent Transportation Systems (Valencia et al., 2019); en esta nueva versión, se obtuvieron mejoras importantes gracias a la implementación del algoritmo de visión artificial You Only Look Once (YOLO), que nos permitió mejorar significativamente la precisión y exactitud de las detecciones tanto de parrilleros como de motociclistas sin casco. 


\section{Sistemas inteligentes de transporte}

Los Sistemas Inteligentes de Transporte (en inglés, ITS) corresponden a la aplicación de tecnologías de la información y comunicación (TIC) para la gestión de los sistemas de transporte convencionales, utilizando mejoras tecnológicas destinadas a optimizar la seguridad, eficiencia, accesibilidad y sostenibilidad del transporte público, sin aumentar la infraestructura existente (Mfenjou et al., 2018). El Ministerio de Transporte (2015) establece que un sistema inteligente de transporte es la combinación de distintas áreas de la ingeniería, tal como: transporte, sistemas, financiera, ambiental, telecomunicaciones, entre otras; en búsqueda de mejorar la eficiencia en el transporte, la mitigación de los impactos ambientales, y salvaguardar la vida humana a través de la seguridad vial.

La inserción de tecnología de punta en el sector transporte se ha afianzado como una de las estrategias más frecuentes utilizada en ciudades como Londres, Barcelona, Nueva York, y Singapur, con el fin de mejorar la movilidad y la seguridad vial de sus pobladores; gracias a los Centros de Gestión de Tráfico (en inglés, TMC) adoptados por estas ciudades, se han relegado los grandes proyectos de infraestructura vial, propios del siglo anterior. Por ello, los ITS pueden asistir a las poblaciones en desarrollo, sirviendo de ayuda para lograr los objetivos deseados en el crecimiento de las mismas (Peláez Valencia, 2016).

\subsection{Tecnologías en los sistemas inteligentes de transporte}

En cuanto a la parte tecnológica, los ITS incorporan un amplio rango de ayuda al usuario, que van desde una simple información de alerta desde un teléfono móvil hasta sistemas sofisticados de control de tránsito (Asociación mundial de la carretera [PIARC], 2016; Sadek, 2016). Para lograrlo, utilizan un amplio grupo de tecnologías disponibles, como las siguientes: tecnologías de procesamiento, gestión y archivo de datos (sistemas de gestión de datos archivados); tecnologías de detección (detectores de tránsito, identificación automática vehicular, detección de velocidad, sensores medioambientales); tecnologías de comunicación (arquitectura de comunicaciones); tecnologías de difusión de la información (carteles de mensajes dinámicos); tecnologías de posicionamiento y localización referencial (sistemas de posicionamiento global); tecnologías de control vehicular y control del tránsito (centros de gestión de tráfico).

\section{Detección automática de matrículas en vehículos}

Desde los primeros años del siglo XX, ha existido un interés creciente en el desarrollo de mecanismos para la seguridad; tecnologías como la identificación biométrica (por ejemplo, usando voz, cara o firma) aparecen como una opción a las huellas digitales. El reconocimiento automático de matrículas (en inglés, ANPR) ha sido estudiado desde 1976 por la policía del Reino Unido (Nguyen et al., 2014). En la totalidad de ciudades del mundo, han ocurrido problemas con el tráfico, accidentes de tránsito, y conductores que rompen la ley; esta clase de problemas ha llevado a la creación de herramientas de identificación y seguimiento para vehículos, y su aplicación se ha extendido a la identificación de vehículos robados (Espinoza y Salinas, 2015). 
El control de vehículos usando sistemas de identificación de matrículas se destaca entre las nuevas tecnologías por su amplia gama de aplicaciones; recientemente ha mejorado la eficiencia de los mismos gracias al uso de cámaras infrarrojas y algoritmos refinados. En el 2003, en Londres se implementó un programa gubernamental para reducir el tráfico en el centro de la ciudad; para lograrlo, se instaló un sistema de reconocimiento de matrículas que identifica los vehículos que ingresan a la zona céntrica y comprueba en la base de datos si han pagado previamente el impuesto estipulado para transitar por la zona antes mencionada, imponiendo una orden de cobro a los infractores detectados. Aunque inicialmente fue una medida temporal, las autoridades británicas decidieron establecerla de forma permanente y extenderla por todo el Reino Unido (Toledo Muñoz, 2005).

\section{Detección de objetos y personas utilizando visión artificial}

Los avances relevantes en la inteligencia artificial han sido posible gracias al desarrollo del aprendizaje automático, que es el uso de algoritmos para el análisis de datos, aprender de estos y luego hacer predicciones sobre algo en el mundo observable (Manrique et al, 2019). Por ello, el uso de clasificadores de imágenes diseñados usando aprendizaje automático supervisado puede resolver el problema de la detección de objetos (Benalcázar, 2019).

El proceso para el reconocimiento automático de objetos inicia con la adquisición de la imagen de una escena en tres dimensiones; a continuación, esta imagen se procesa para mejorar su calidad y eliminar posibles imperfecciones; el siguiente paso consiste en separar el objeto de interés del fondo, seguido de la extracción de las características que describen al objeto (color, textura y geometría); finalmente, se comparan dichas características con las de otros objetos ubicados en la base de conocimiento, y de esa forma determinar el tipo de objeto (García Santillán, 2008).

Las características más empleadas en el campo de la visión por computadora son: la transformada de Hough; los patrones binarios locales (en inglés, LBP); el histograma de gradientes orientados (en inglés, HOG); y la transformación de la característica invariante de escala (en inglés, SIFT) (Dalal y Triggs, 2005). Chiverton (2012) realizó el primer sistema automatizado propuesto y probado para la detección de motociclistas que conducían sin casco; este sistema utiliza un clasificador support vector machine (SVM), entrenado en características derivadas de datos de imagen cerca de la región principal de los motociclistas. Las características seleccionadas capturan la forma y la propiedad reflectante de los cascos, donde la mitad superior de la superficie es más brillante que la mitad inferior. El sistema también tiene en cuenta la forma de arco circular del casco, para lo cual usa una técnica de detección basada en la transformada de Hough desarrollada por Pei y Horng (1995).

Sin embargo, este enfoque conduce a una gran cantidad de errores de clasificación, ya que algunos objetos parecidos a los cascos se clasifican como tal, mientras algunos cascos que son diferentes no son clasificados. Otra limitación es la no identificación primaria de los motociclistas en el cuadro, lo cual debe hacerse ya que el casco solo es relevante en el caso de los motociclistas. 
Para superar el problema de la clasificación errónea, crearon un sistema que identifica, en primer lugar, a los motociclistas en el cuadro empleando un clasificador SVM entrenado en características extraídas por el descriptor LBP. Luego, la clasificación del casco se realiza utilizando SVM en las características extraídas por un descriptor híbrido, que se crea mediante la combinación de la transformada circular de Hough, y los descriptores HOG y LBP (Silva et al., 2013).

\section{Materiales y métodos}

En este trabajo, se adoptó la versión actualizada de la metodología de investigación científica de diseño en sistemas de información (Vaishnavi et al., 2004), compuesta por las siguientes fases: conocimiento del problema; sugerencia; desarrollo; y evaluación.

\subsection{Conocimiento del problema}

Mediante la revisión de literatura se evidenció el problema creciente que está generando el transporte informal, específicamente fenómenos como el "mototaxismo", en Colombia. Por otra parte, encontramos la insuficiencia económica existente para implementar por completo la normativa de los Sistemas Estratégicos de Transporte Público (SETP), los cuales fueron reglamentados hace 10 años, pero muchas de las ciudades intermedias colombianas no han adoptado debido a los altos costos que conlleva (Ramirez et al, 2019).

\subsection{Sugerencia}

Se estudiaron diferentes alternativas de diseños para satisfacer la necesidad encontrada, manteniendo costos de implementación bajos y cumpliendo la normativa colombiana, la cual indica que no está permitido el uso de vehículos en movimiento como herramientas para foto-detección (Ministerio de Transporte, 2018b). La Tabla 1 muestra el cumplimiento de requerimientos para dos tipos de herramientas estudiados (fijos y móviles), basados en la legislación colombiana.

\begin{tabular}{llllll}
\hline & \multicolumn{6}{c}{ Requerimientos } \\
\cline { 2 - 6 } $\begin{array}{l}\text { Tipo de } \\
\text { herramienta }\end{array}$ & $\begin{array}{l}\text { Criterios } \\
\text { técnicos }\end{array}$ & $\begin{array}{l}\text { Mecanismos } \\
\text { de calibración }\end{array}$ & $\begin{array}{l}\text { Set de } \\
\text { pruebas } \\
\text { de } \\
\text { software }\end{array}$ & $\begin{array}{l}\text { Autorización } \\
\text { del ministerio } \\
\text { de transporte }\end{array}$ & $\begin{array}{l}\text { Señalización } \\
\text { 5oo metros } \\
\text { antes }\end{array}$ \\
\hline Fija & $x$ & $X$ & $x$ & $x$ & $X$ \\
\hline Móvil & $\checkmark$ & $\checkmark$ & $\checkmark$ & $\checkmark$ & $\checkmark$ \\
\hline
\end{tabular}

Tabla 1 - Cumplimiento de los requerimientos normativos exigidos por la ley colombiana por herramienta de detección automática

Se diseñó el sistema presentado en este trabajo, usando la cámara de un teléfono móvil ubicado sobre un trípode y una aplicación, como herramienta para el monitoreo de las infracciones cometidas por los motociclistas en puntos estratégicos de la ciudad, donde 
se ubican los oficiales de tránsito. Acorde con la reglamentación, los oficiales pueden usar este tipo de herramientas tecnológicas para detectar infracciones, encontrando ejemplos como los detectores de velocidad y el alcoholímetro.

\subsection{Desarrollo}

La aplicación móvil fue desarrollada con herramientas de desarrollo libres; se usó OpenCV, una librería de visión por computador, para el procesamiento de la imagen y la localización del área de la matrícula. También se usó Tesseract, una librería de reconocimiento óptico de caracteres (en inglés, OCR), para reconocer los caracteres de las matrículas. La Figura 1 ilustra el diseño conceptual planteado para el sistema.

En una versión preliminar a este trabajo, para la detección de casco y pasajeros se usó exclusivamente la función imfindcircles en MATLAB (Valencia et al., 2019); esta función usa la transformada circular de Hough para detectar círculos analizando las imágenes. Para esto se calibraron los parámetros de sensibilidad y luminosidad con el fin de lograr detectar los cascos en los motociclistas.

Ahora, con el fin de mejorar la precisión y exactitud en la detección de cascos y parrilleros, se usó el algoritmo de inteligencia artificial entrenado para la detección de objetos, YOLO. Posteriormente, procedemos a comparar los resultados obtenidos en la detección de casco con los resultados obtenidos realizando la misma tarea con la transformada circular de Hough.

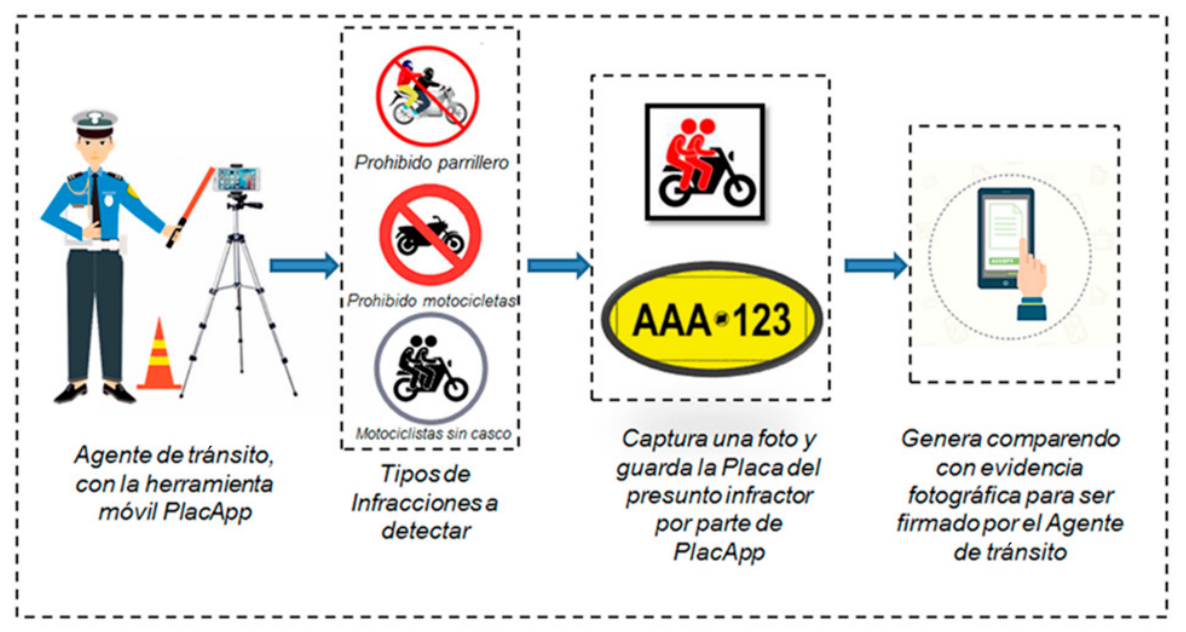

Figura 1 - Diseño del sistema automático de detección compuesto por un teléfono móvil, aplicación móvil, batería portátil para teléfono móvil y trípode para teléfono móvil

\subsection{Características de la prueba}

Las pruebas experimentales del sistema se realizaron en la ciudad de Valledupar, Colombia (latitud 10.474621, longitud -73.248559), entre 11:00 am y 12:00 m con clima 
despejado; a causa de la autonomía energética del equipo empleado, las pruebas tuvieron una duración total de una hora. Se utilizó un teléfono móvil de referencia Motorola Moto G4, el cual posee una cámara de 13 megapíxeles; este dispositivo fue instalado en un trípode de $70 \mathrm{~cm}$ de altura. El equipo fue ubicado en la calle 17 con carrera $9^{\mathrm{a}}$, una intersección semafórica de alto flujo vehicular en la principal zona comercial de la ciudad, con el fin de capturar la mayor cantidad de imágenes posibles. Se tomaron 105 imágenes de motociclistas mientras conducían a velocidades entre 20 y $50 \mathrm{~km} / \mathrm{h}$. El sistema permite una buena visibilidad tanto de las matrículas como de las personas que van en las motocicletas. La Figura 2 muestra la detección y reconocimiento de una matrícula a través de la aplicación móvil; en este caso, los caracteres de la matrícula fueron reconocidos de forma correcta.

\section{Validación experimental}

Con el fin de validar el prototipo desarrollado durante este trabajo, uno de los investigadores evaluó cada una de las imágenes capturadas en la prueba mediante una verificación visual para identificar sus características reales y las posibles infracciones presentes en estas. Empleando las Ecuaciones 1 y 2 (Kulkarni et al., 2018) se calcularon los porcentajes de exactitud y precisión de las pruebas realizadas; en estas ecuaciones, $p$ es el número de muestras clasificadas correctamente, $q$ es el número de muestras clasificadas incorrectamente, y $N$ es el número total de muestras.

$$
\begin{gathered}
\text { Exactitud }=\frac{p}{N} \\
\text { Precisión }=\frac{p}{N+q}
\end{gathered}
$$

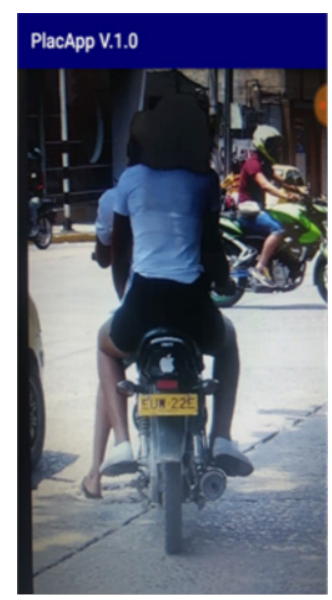

(a)

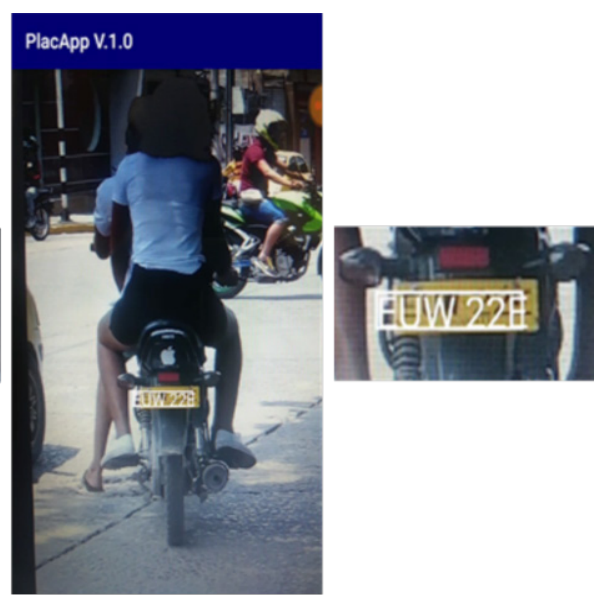

(b)

Figura 2 - (a) Matrícula sin reconocimiento;

(b) Reconocimiento de matrícula hecho por la aplicación móvil 
Para concluir esta investigación, consideraremos el problema en contexto con los aspectos socioculturales colombianos, la legislación nacional, la hipótesis planteada, los resultados y la evaluación de la solución desarrollada. En Universidad EAFIT (s. f.) se puede acceder al repositorio con reportes técnicos, ponencias, e información detallada de este trabajo.

\section{Resultados}

Durante las pruebas realizadas en la ciudad de Valledupar se detectaron 40 matrículas de motocicletas. La exactitud fue de 85,5\%, y la precisión del 77,7\%. El prototipo no pudo reconocer 5 de las 40 matrículas capturadas durante las pruebas; este error es causado por factores ambientales (sobreexposición a la luz) o por la posición de la matrícula, ya que su ángulo - respecto a la cámara del móvil- no es el ideal para la correcta detección. La Figura 3 muestra dos ejemplos relacionados con estos casos.

Con la implementación de YOLO y la integración de la transformada circular de Hough en las pruebas, se analizaron 65 imágenes para identificar la presencia de cascos y parrillero, donde se obtuvo una precisión del 58\% y una exactitud del $73,1 \%$ en la detección de cascos; en el caso de detección de parrilleros, se obtuvo una precisión y exactitud de $87,5 \%$ y $77,7 \%$ respectivamente. Las Figuras 4 y 5 son un ejemplo de los avances logrados.

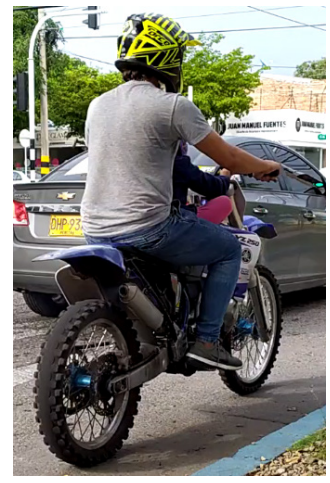

(a)

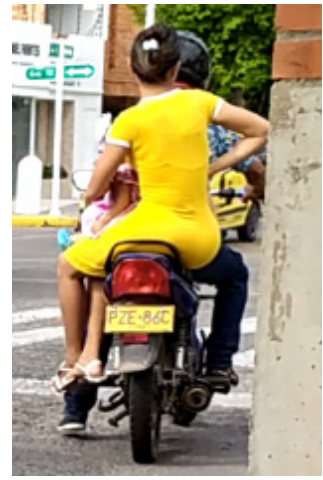

(b)

Figura 3 - (a) Matrícula con un ángulo que no permite el reconocimiento;

(b) Matrícula con sobreexposición a la luz

Una de las pruebas que se hizo fue usar la transformada de Hough para la detec-ción del casco en una imagen donde previamente se había realizado la detección de pasajero con YOLO, para comprobar si delimitando el área de la imagen se reduce el error en la detección del casco. Las Tablas 2 y 3 corresponden a las matrices de confusión de los problemas de detección de cascos y parrilleros usando la transformada circular de Hough. Del mismo modo, las Tablas 4 y 5 corresponden a las matrices de confusión para los dos casos mencionados usando YOLO. 


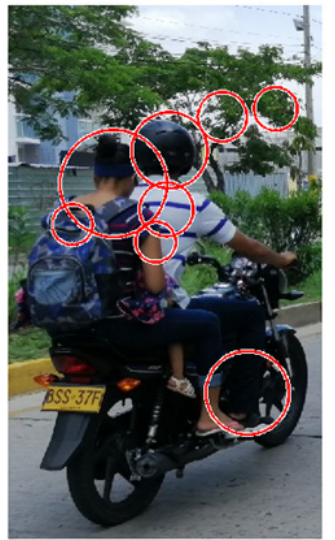

(a)

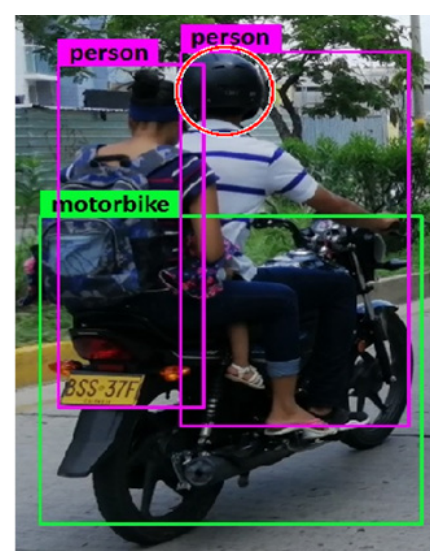

(b)

Figura 4 - (a) Detección de casco con la transformada circular de Hough;

(b) Detección de casco con YOLO y la transformada circular de Hough

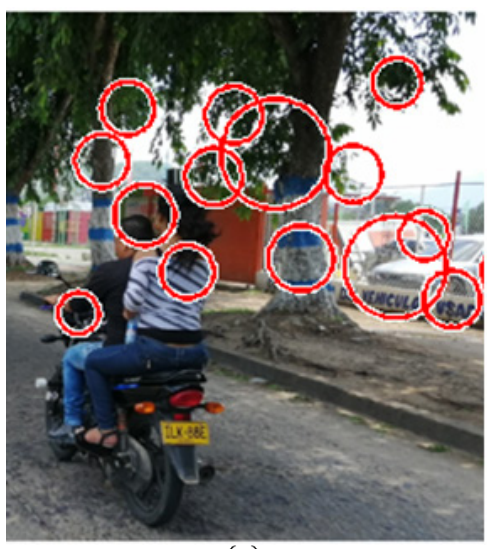

(a)

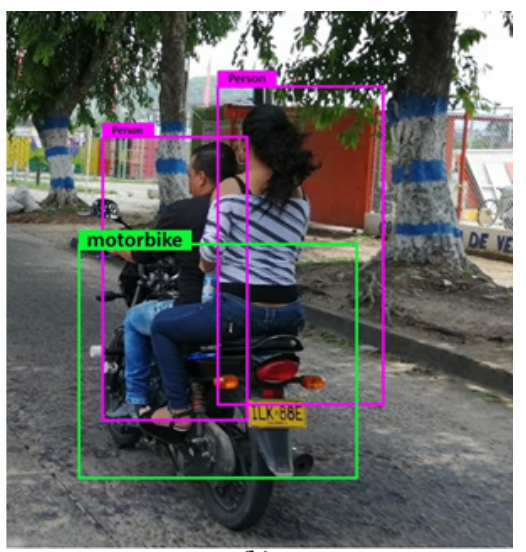

(b)

Figura 5 - (a) Detección de parrillero con la transformada circular de Hough;

(b) Detección de parrillero con YOLO

\begin{tabular}{|c|c|c|c|}
\hline & & \multicolumn{2}{|c|}{ Valor predicho } \\
\hline & & Con casco & Sin casco \\
\hline \multirow{2}{*}{$\begin{array}{c}\text { Valor } \\
\text { real }\end{array}$} & Con casco & 32 & 8 \\
\hline & Sin casco & 15 & 10 \\
\hline
\end{tabular}

Tabla 2 - Matriz de confusión para detección de casco con la transformada circular de Hough 


\begin{tabular}{cccc} 
& & \multicolumn{3}{c}{ Valor predicho } \\
\cline { 3 - 4 } Valor & Con parrillero & Con parrillero & Sin parrillero \\
\cline { 3 - 4 } real & Sin parrillero & 9 & 11 \\
\cline { 3 - 4 } & & 1 & 19 \\
\hline
\end{tabular}

Tabla 3 - Matriz de confusión para detección de parrillero con la transformada circular de Hough

\begin{tabular}{|c|c|c|c|}
\hline & & \multicolumn{2}{|c|}{ Valor predicho } \\
\hline & & Con casco & Sin casco \\
\hline \multirow{2}{*}{$\begin{array}{l}\text { Valor } \\
\text { real }\end{array}$} & Con casco & 34 & 6 \\
\hline & Sin casco & 11 & 14 \\
\hline
\end{tabular}

Tabla 4 - Matriz de confusión para detección de casco con YOLO y la transformada circular de Hough

\begin{tabular}{cccc} 
& & \multicolumn{2}{c}{ Valor predicho } \\
\cline { 3 - 4 } Valor & Con parrillero & Con parrillero & Sin parrillero \\
\cline { 3 - 4 } real & Sin parrillero & 16 & 4 \\
\cline { 3 - 4 } & & 1 & 19 \\
\hline
\end{tabular}

Tabla 5 - Matriz de confusión para detección de parrillero con YOLO

\section{Conclusiones}

Las autoridades municipales de las ciudades intermedias en Colombia tienen un gran interés en incrementar la seguridad vial; en particular, buscan reducir los accidentes de tránsito causados por motocicletas, ya que se ha demostrado que este medio de transporte tiene un alto índice de accidentalidad. Con este trabajo, proponemos un nuevo sistema para monitorear y controlar el transporte ilegal con motocicletas, el cual que está altamente relacionado con accidentes viales.

A través de las pruebas realizadas, confirmamos la hipótesis inicial: usando una aplicación móvil, logramos reconocer las matrículas de motocicletas, y empleando la transformada circular de Hough en MATLAB se logró detectar cuando los motociclistas portaban el casco. La precisión fue del $77 \%$ y la exactitud fue del $85,5 \%$ para el caso de reconocimiento de matrículas.

La precisión para detectar el casco y el parrillero haciendo uso exclusivo de la transformada circular de Hough fue del 53\% y el $67 \%$, respectivamente; cuando se implementó YOLO junto con la transformada circular de Hough, tanto la precisión como la exactitud aumentaron a 67,9\% y $80 \%$, demostrando que si es posible mejorar el sistema de detección -con base en estos parámetros- gracias a los algoritmos de inteligencia artificial entrenados para la detección de objetos. 
Las limitaciones encontradas en el prototipo desarrollado se presentan cuando hay presencia de luz solar en exceso, en cuyo caso las matrículas de las motocicletas quedan sobreexpuestas en las fotos. Otra limitación es que el sistema no está diseñado para trabajar 24 horas, 7 días a la semana. Para mejorarlo, se requiere de hardware especializado.

El ideal es implementar los algoritmos de inteligencia artificial en la aplicación móvil de forma directa, con el fin de evitar el uso de MATLAB en una siguiente versión. Como trabajo futuro, proponemos entrenar los algoritmos de detección automática de matrículas para mejorar su precisión, además de crear un clasificador usando YOLO para la detección de cascos, en busca entrenar el algoritmo para que logre identificarlos y así optimizar el método de detección por medio de circunferencias.

\section{Agradecimientos}

Este estudio fue financiado por el Ministerio de Ciencia, Tecnología e Innovación MINCIENCIAS, Colombia (Concesión No. 111572553478) en el marco del programa Jóvenes Investigadores e Innovadores (Contrato No. FP44842-301-2018). Los autores extienden su agradecimiento a la Vicerrectoría de Descubrimiento y Creación de la Universidad EAFIT, y a la Gobernación del Cesar.

\section{Referencias}

Alcaldía de Valledupar. (2018, 29 de mayo). Decreto 558 de 2018 "Por medio del cual se adopta medidas tendientes a reglamentar el transporte en motocicletas, se promueve la seguridad vial y se dictan otras disposiciones".

Asociación mundial de la carretera. (2016). Manual Explotación de la Red Vial \& Sistemas Inteligentes de Transporte: Guía para profesionales. Retreived from: https://rno-its.piarc.org/es

Association des Constructeurs Européens de Motocycles. (2009). MAIDS: In-depth investigations of accidents involving powered two wheelers- Final Report 2.o. Retreived from: http://www.maids-study.eu/pdf/MAIDS2.pdf

Benalcázar, M. E. (2019). Machine Learning for Computer Vision: a review of Theory and Algorithms. RISTI - Revista Ibérica de Sistemas e Tecnologias de Informação, (E19), 608-618. Retreived from: http://www.risti.xyz/issues/ristie19.pdf

Chiverton, J. (2012). Helmet presence classification with motorcycle detection and tracking. IET Intelligent Transport Systems, 6(3), 259-269. DOI: https://doi. org/10.1049/iet-its.2011.0138

Dalal, N., \& Triggs, B. (2005). Histograms of oriented gradients for human detection. En 2005 IEEE Computer Society Conference on Computer Vision and Pattern Recognition (CVPR'05), (pp. 886-893). IEEE. DOI: https://doi.org/10.1109/ CVPR.2005.177 
Doval, P. (2017, 7 de mayo). India is now the world's biggest two-wheeler market. The Times of India. Retreived from: https://timesofindia.indiatimes.com/auto/bikes/ india-is-now-worlds-biggest-2-wheeler-market/articleshow/58555735.cms

Espinoza, D., \& Salinas, C. (2015). Desarrollo de un Sistema de Reconocimiento de Placas Vehiculares (tesis de pregrado, Universidad del Azuay). Repositorio Institucional Universidad del Azuay. Retreived from: http://dspace.uazuay.edu. ec/handle/datos/5047

García Santillán, E. (2008). Detección y clasificación de objetos dentro de un salón de clases empleando técnicas de procesamiento digital de imágenes (tesis de maestría, Universidad Autónoma Metropolitana). Repositorio Institucional UAM. Retreived from: http://newton.azc.uam.mx/mcc/o1_esp/11_tesis/tesis/terminada/o80513_ garcia_santillan_elias.pdf

Gwilliam, K. M. (2002). Cities on the move: a World Bank urban transport strategy review. World Bank Group. Retreived from: http://documents.worldbank.org/ curated/en/928301468762905413/Cities-on-the-Move-A-World-Bank-UrbanTransport-Strategy-Review

Kulkarni, Y., Kamthe, A., Bodkhe, S., \& Patil, A. (2018). Automatic Number Plate Recognition for Motorcyclists Riding Without Helmet. En 2018 International Conference on Current Trends towards Converging Technologies (ICCTCT). (pp. 1-6). IEEE. DOI: https://doi.org/10.1109/ICCTCT.2018.8551001

Manrique, R., Amézquita, N., \& Carreño, J. P. (2019). Oportunidades y desafíos en la aplicación de Inteligencia Artificial a procesos de Validación Marcaria. RISTI - Revista Ibérica de Sistemas e Tecnologias de Informação, (E24), 221-233. Retreived from: http://www.risti.xyz/issues/ristie24.pdf

Mfenjou, M. L., Abba Ari, A. A., Abdou, W., Spies, F., \& Kolyang. (2018). Methodology and trends for an intelligent transport system in developing countries. Sustainable Computing: Informatics and Systems, 19, 96-111. DOI: https://doi.org/10.1016/j. suscom.2018.08.002

Ministerio de Transporte. (2015, octubre 22). Decreto 2060 de 2015 "Por el cual se adiciona el Decreto 1079 de 2015 y se reglamenta el artículo 84 de la Ley 1450 de 2011".

Ministerio de Transporte. (2018). Unidad de movilidad urbana sostenible: Informe Ejecutivo 2010-2018. Retreived from: https://www.mintransporte.gov.co/ descargar.php?id=6698

Ministerio de Transporte. (2018, 22 de marzo). Resolución 718 de 2018 "Por la cual se reglamentan los criterios técnicos para la instalación y operación de medios técnicos o tecnológicos para la detección de presuntas infracciones al tránsito y se dictan otras disposiciones". 
Nguyen, T., Nguyen, D., \& Nguyen, P. (2014). UIT-ANPR: Toward an open framework for automatic number plate recognition on smartphones. En Proceedings of the 8th International Conference on Ubiquitous Information Management and Communication (ICUIMC'14). (pp. 1-5). Association for Computing Machinery. DOI: https://doi.org/10.1145/2557977.2558037

Observatorio Nacional de Seguridad Vial. (2019). Cifras definitivas enero - diciembre 2018: Reporte Diciembre de 2018. Retreived from: https://ansv.gov.co/ observatorio/index4b78.html?op $=$ Contenidos\&sec $=59$

Pei, S. C., \& Horng, J. H. (1995). Circular arc detection based on Hough transform. Pattern Recognition Letters, 16(6), 615-625. DOI: https://doi.org/10.1016/01678655(95)80007-G

Peláez Valencia, A. M. (2016). Modelo de cuantificación económica para implementación de Sistemas Inteligentes de Transporte (ITS) en Medellín (tesis de maestría, Universidad Nacional de Colombia). Repositorio Institucional UN. Retreived from: https://repositorio.unal.edu.co/handle/unal/57742

Ramirez, T., Castañeda, L., \& Toro, M. (2019). Sistema inteligente para transporte público tipo SETP. En A. M. Vélez-Pereira y D. Toro González (Eds.), XIII Congreso Colombiano de Transporte y Tránsito: Movilizando el futuro de Co-lombia (p. 57). Universidad Tecnológica de Bolívar.

Rohith, C. A., Nair, S. A., Nair, P. S., Alphonsa, S., \& John, N. P. (2019). An efficient helmet detection for MVD using deep learning. En 2019 3rd International Conference on Trends in Electronics and Informatics (ICOEI), (pp. 282-286). IEEE. DOI: https://doi.org/10.1109/icoei.2019.8862543

Sadek, A. (2016). Tecnologías ITS. PIARC. Retreived from: https://rno-its.piarc.org/ es/conceptos-basicos-its/tecnologias-its

Silva, R., Aires, K., \& Veras, R. (2014). Helmet Detection on Motorcyclists Using Image Descriptors and Classifiers. En 2014 27th SIBGRAPI Conference on Graphics, Patterns and Images, (pp. 141-148). IEEE. DOI: https://doi.org/10.1109/ SIBGRAPI.2014.28

Silva, R., Aires, K., Santos, T., Abdala, K., Veras, R., \& Soares, A. (2013). Automatic detection of motorcyclists without helmet. En 2013 XXXIX Latin American Computing Conference (CLEI). (pp. 1-7). IEEE. DOI: https://doi.org/10.1109/ CLEI.2013.6670613

Tobón, A., \& Galvis, D. (2009). Análisis sobre la evolución reciente del sector de transporte en Colombia. Perfil de coyuntura económica, (13), 147-163. Retreived from: http://hdl.handle.net/10495/6754

Toledo Muñoz, L. E. (2005). Reconocimiento automático de matrículas de automóvil [tesis de maestría, Centro de Investigadores en Óptica]. Repositorio Institucional CIO. Retreived from: https://biblioteca.cio.mx/tesis/10857.pdf 
Universidad EAFIT (s. f.) Plataforma inteligente para sistemas estratégicos de transporte público (SETP). Consultado el 25 de mayo de 2020. Retreived from: http://www.eafit.edu.co/investigacion/grupos/gemi/proyectos/Paginas/ plataforma-inteligente-para-sistemas-estrategicos-de-transporte-publicosetp.aspx

Vaishnavi, V., Kuechler, B., \& Petter, S. (2004). Design Science Research in Information Systems. Última actualización: 30 de junio de 2019. Retreived from: http://www.desrist.org/design-research-in-information-systems/

Valencia, J. F., Ramirez-Guerrero, T., Castañeda, L. F., \& Toro, M. (2019). Automatic detection of number-plate and traffic infractions of motorcyclists by Intelligent Transportation Systems (manuscrito presentado para publicación). Escuela de Ingeniería, Universidad EAFIT.

Waranusast, R., Bundon, N., Timtong, V., Tangnoi, C., \& Pattanathaburt, P. (2013). Machine vision techniques for motorcycle safety helmet detection. En 2013 28th International Conference on Image and Vision Computing New Zealand (IVCNZ 2013). (pp. 35-40). IEEE. DOI: https://doi.org/10.1109/IVCNZ.2013.6726989 